\title{
Pattern Dynamics in a Spatial Predator-Prey System with Allee Effect
}

\author{
Gui-Quan Sun, ${ }^{1,2,3,4}$ Li Li, ${ }^{5}$ Zhen Jin, ${ }^{1,2}$ Zi-Ke Zhang, ${ }^{3}$ and Tao Zhou ${ }^{6}$ \\ ${ }^{1}$ Complex Sciences Center, Shanxi University, Taiyuan, Shan'xi 030006, China \\ ${ }^{2}$ School of Mathematical Sciences, Shanxi University, Taiyuan, Shan'xi 030006, China \\ ${ }^{3}$ Institute of Information Economy, Hangzhou Normal University, Hangzhou 310036, China \\ ${ }^{4}$ Department of Mathematics, North University of China, Taiyuan, Shan'xi 030051, China \\ ${ }^{5}$ Department of Mathematics, Taiyuan Institute of Technology, Taiyuan, Shan'xi 030008, China \\ ${ }^{6}$ Web Sciences Center, University of Electronic Science and Technology of China, Chengdu, Sichuan 610054, China \\ Correspondence should be addressed to Gui-Quan Sun; gquansun@126.com
}

Received 9 May 2013; Accepted 22 August 2013

Academic Editor: Rasajit Bera

Copyright (C) 2013 Gui-Quan Sun et al. This is an open access article distributed under the Creative Commons Attribution License, which permits unrestricted use, distribution, and reproduction in any medium, provided the original work is properly cited.

\begin{abstract}
We investigate the spatial dynamics of a predator-prey system with Allee effect. By using bifurcation analysis, the exact Turing domain is found in the parameters space. Furthermore, we obtain the amplitude equations and determine the stability of different patterns. In Turing space, it is found that predator-prey systems with Allee effect have rich dynamics. Our results indicate that predator mortality plays an important role in the pattern formation of populations. More specifically, as predator mortality rate increases, coexistence of spotted and stripe patterns, stripe patterns, spotted patterns, and spiral wave emerge successively. The results enrich the finding in the spatial predator-prey systems well.
\end{abstract}

\section{Introduction}

The Allee effect, named after the ecologist Warder Clyde Allee, has been recognized as an important phenomenon of positive density dependence in low-density population [1-5]. Allee effect can occur whenever fitness of an individual in a small or sparse population decreases as the population size or density also declines $[6,7]$. Since the outstanding work of Allee [1], the Allee effect has been regarded as one of the central and highly important issues in the population and community ecology. And its critical importance has widely been realized in the conservation biology that Allee effect is most likely to increase the extinction risk of low-density populations. As a result, studies on Allee effect have received more and more attention from both mathematicians and ecologists.

Long time series of the density of both prey and predator is needed, so it is difficult to analyse their dynamics. As a result, it may provide useful information by constructing mathematical models to investigate the dynamical behaviors of predator-prey systems. There have been a large group of papers on predator-prey systems with Allee effect [8-13].
However, these previous works did not take into account the effect of space.

There are also some works done on spatial predatorprey systems with Allee effect [14-16]. Petrovskii et al. found that the deterministic system with Allee effect can induce patch invasion [14]. Morozov et al. found that the temporal population oscillations can exhibit chaotic dynamics even when the distribution of the species in the space was regular [15]. Moreover, they found that the chaos accompanied with patch invasion even though the environments were heterogeneous [16]. However, their results were obtained by choosing particular initial conditions. Then, it is natural to ask what kind of patterns can be obtained in predator-prey systems with Allee effect by using other initial conditions. To understand that mechanism well, we will investigate a predator-prey system with Allee effect.

Because of the insightful work of many scientists over recent years, we can make research on pattern selection by using the standard multiple scale analysis $[17,18]$, in which the control parameters and the derivatives are expanded in terms of a small enough parameter. In the neighborhood of 
the bifurcation points (Hopf and Turing bifurcation points), the critical amplitudes follow the normal forms, and thus their general forms can be obtained from the methods of symmetry-breaking bifurcations.

The paper is organized as follows. In Section 2, we present a predator-prey system with Allee effect and give Turing region in parameters space. In Section 3, by using multiple scale analysis, we obtain amplitude equations. In Section 4, we show the spatial patterns by a series of numerical simulations. Finally, conclusions and discussions are presented in Section 5.

\section{A Predator-Prey System with Allee Effect}

We consider the following model of two-dimensional spatiotemporal system [14-16, 19]:

$$
\begin{aligned}
& \frac{\partial H}{\partial T}=F(H)-f(H, P)+D_{1} \Delta H, \\
& \frac{\partial P}{\partial T}=\kappa f(H, P)-D(P)+D_{2} \Delta P,
\end{aligned}
$$

where $H=H(X, Y, T)$ and $P=P(X, Y, T)$ are densities of prey and predator, respectively, at time $T$ and position $(X, Y)$. The function $F(H)$ represents the intrinsic prey growth, $f(H, P)=f(H) P$ represents predation term, $\kappa$ is the food utilization coefficient, $D_{1}$ and $D_{2}$ are diffusion coefficients, and $D(P)$ describes predator mortality.

It is assumed that the predation term is a bilinear form of prey and predator density and predator mortality is a nonlinear function of predator density. As a result, we choose $f(H, P)=H P$ and $D(P)=M P^{2}[20]$.

When the prey population obeys Allee dynamics, its growth rate can be parameterized as follows $[14,15,21]$ :

$$
F(H)=\frac{4 \omega}{\left(K-H_{0}\right)^{2}} H\left(H-H_{0}\right)(K-H),
$$

where $K$ is the prey-carrying capacity, $\omega$ is the maximum per capita growth rate, and $H_{0}$ quantifies the intensity of the Allee effect. If $0<H_{0}<K, F(H)$ is a strong Allee effect; if $-K<H_{0}<0, F(H)$ is a weak Allee effect; if $H_{0} \leq-K$, the Allee effect is absent.

In order to minimize the number of parameters involved in the model system, it is extremely useful to write the system in a nondimensionalized form. Although there is no unique method of doing this, it is often a good idea to relate the variables to some key relevant parameters. Introducing dimensionless variables

$$
\begin{aligned}
& u=\frac{H}{K}, \quad v=\frac{P}{\kappa K}, \quad t=a T, \\
& \bar{X}=X \sqrt{\frac{a}{D_{1}}}, \quad \bar{Y}=Y \sqrt{\frac{a}{D_{1}}},
\end{aligned}
$$

we obtain the following equations:

$$
\begin{gathered}
\frac{\partial u}{\partial t}=\gamma u(u-\beta)(1-u)-u v+\Delta u \\
\frac{\partial v}{\partial t}=u v-\delta v^{2}+\varepsilon \Delta v
\end{gathered}
$$

where

$$
\begin{array}{ll}
\beta=\frac{H_{0}}{K}, & \gamma=\frac{4 \omega K}{A \kappa\left(K-H_{0}\right)^{2}}, \\
\delta=\frac{M}{a}, & \varepsilon=\frac{D_{2}}{D_{1}} .
\end{array}
$$

First of all, we need to investigate the dynamics of nonspatial model of systems (4a) and (4b)

$$
\begin{gathered}
\frac{d u}{d t}=\gamma u(u-\beta)(1-u)-u v \\
\frac{d v}{d t}=u v-\delta v^{2}
\end{gathered}
$$

Systems (6a) and (6b) have three boundary equilibrium named $E_{0}=(0,0), E_{1}=(1,0)$, and $E_{2}=(\beta, 0)$ and two interior equilibriums named $E_{3}$ and $E_{4}$, where

$$
\begin{aligned}
& E_{3}=\left(\frac{\gamma \delta+\gamma \beta \delta-1+\sqrt{Q}}{2 \gamma \delta}, \frac{\gamma \delta+\gamma \beta \delta-1+\sqrt{Q}}{2 \gamma \delta^{2}}\right), \\
& E_{4}=\left(\frac{\gamma \delta+\gamma \beta \delta-1-\sqrt{Q}}{2 \gamma \delta}, \frac{\gamma \delta+\gamma \beta \delta-1-\sqrt{Q}}{2 \gamma \delta^{2}}\right),
\end{aligned}
$$

where $Q=(\gamma \delta)^{2}-2(\gamma \delta)^{2} \beta-2 \gamma \beta \delta+1$.

From a biological point of view, we are concerned with the dynamics of $E_{3}$ and $E_{4}$. The Jacobian matrix corresponding to the equilibrium point is that

$$
J=\left(\begin{array}{ll}
a_{11} & a_{12} \\
a_{21} & a_{22}
\end{array}\right)
$$

where

$$
\begin{gathered}
a_{11}=2 \gamma u^{*}-\gamma \beta-3 \gamma\left(u^{*}\right)^{2}+2 \gamma \beta u^{*}, \\
a_{12}=-u^{*}, \\
a_{21}=v^{*}-\delta, \\
a_{22}=u^{*} .
\end{gathered}
$$

Diffusion-driven instability requires the stable, homogeneous steady state is driven unstable by the interaction of the dynamics and diffusion of the species; and therefore

$$
\begin{gathered}
a_{11}+a_{22}<0, \\
a_{11} a_{22}-a_{12} a_{21}>0 .
\end{gathered}
$$


It is found from direct calculations that $E_{3}$ is unstable and $E_{4}$ is stable. Denote $E_{4}=\left(u^{*}, v^{*}\right)$.

Following the standard linear analysis of the reactiondiffusion equation [22], we consider a perturbation near the steady state:

$$
\begin{aligned}
& u(\vec{r}, t)=u^{*}+\bar{u}(r, t), \\
& v(\vec{r}, t)=v^{*}+\bar{v}(r, t),
\end{aligned}
$$

where $\bar{u}(r, t) \ll u^{*}, \bar{v}(r, t) \ll v^{*}$, and $r=(\bar{X}, \bar{Y})$. Assume that

$$
\left(\begin{array}{l}
\bar{u}(r, t) \\
\bar{v}(r, t)
\end{array}\right)=\left(\begin{array}{c}
\alpha_{1} \\
\alpha_{2}
\end{array}\right) e^{\lambda t} e^{i\left(\kappa_{X} X+\kappa_{Y} Y\right)},
$$

where $\lambda$ is the growth rate of perturbation in time $t, \alpha_{1}$ and $\alpha_{2}$ represent the amplitudes, and $\kappa_{X}$ and $\kappa_{Y}$ are the wave number of the solutions.

The characteristic equation of the systems (4a) and (4b) is

$$
(A-\lambda I)\left(\begin{array}{c}
\bar{u} \\
\bar{v}
\end{array}\right)=0
$$

where

$$
A=\left(\begin{array}{cc}
a_{11}-\left(\kappa_{X}^{2}+\kappa_{Y}^{2}\right) & a_{12} \\
a_{21} & a_{22}-\varepsilon\left(\kappa_{X}^{2}+\kappa_{Y}^{2}\right)
\end{array}\right) .
$$

As a result, we have characteristic polynomial:

$$
\begin{gathered}
\lambda^{2}-t r_{\kappa} \lambda+\Delta_{\kappa}=0, \\
t r_{\kappa}=a_{11}+a_{22}-\kappa^{2}(1+\varepsilon) \stackrel{\Delta}{=} t r_{J}-\kappa^{2}(1+\varepsilon), \\
\Delta_{\kappa}=a_{11} a_{22}-a_{12} a_{21}-\kappa^{2}\left(a_{11} \varepsilon+a_{22}\right)+\kappa^{4} \varepsilon \\
\stackrel{\Delta}{=} \Delta_{J}-\kappa^{2}\left(a_{11} \varepsilon+a_{22}\right)+\kappa^{4} \varepsilon
\end{gathered}
$$

where $\kappa^{2}=\kappa_{X}^{2}+\kappa_{Y}^{2}$.

The roots of (15) can be obtained by the following form:

$$
\lambda_{\kappa}=\frac{1}{2}\left(t r_{\kappa} \pm \sqrt{t r_{\kappa}^{2}-4 \Delta_{\kappa}}\right) .
$$

When $\operatorname{Im}\left(\lambda_{\kappa}\right) \neq 0$ and $\operatorname{Re}\left(\lambda_{\kappa}\right)=0$, Hopf bifurcation will emerge. Then, we have that the critical value of Hopf bifurcation parameter- $\delta$ equals

$$
\delta_{H}=\frac{\gamma(\gamma+\beta-1)}{\gamma^{2} \beta^{2}+\gamma^{2}-2 \gamma^{2} \beta-1} .
$$

When $\kappa^{2}=\left(\kappa_{T}\right)^{2}=\sqrt{\Delta_{J} / \varepsilon}$ and $\operatorname{Im}\left(\lambda_{\kappa}\right)=0, \operatorname{Re}\left(\lambda_{\kappa}\right)=0$, Turing bifurcation will occur. Denote $\delta_{T}$ as the critical value of $\delta$ as Turing instability occurs. Since the expression is complicated, we omit it here.

In Figure 1, we show the two critical lines in the parameter space spanned by $\beta$ and $\delta$. The equilibria that can be found in the region, marked by $T$ (Turing space), are stable with

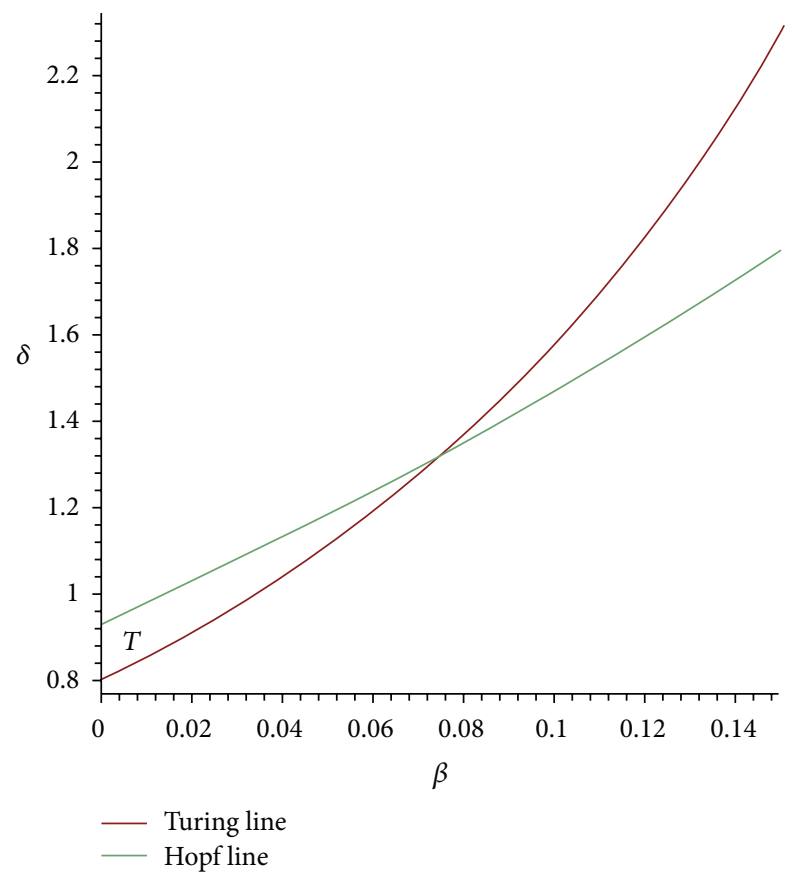

Figure 1: Bifurcation diagram for the systems (4a) and (4b). The green one is the Hopf bifurcation critical line and the red one, Turing bifurcation critical line. The figure shows the Turing space which is marked by $T$. Parameters values: $\gamma=1.5$ and $\varepsilon=0.15$.

respect to the homogeneous perturbations, but they lose their stability with respect to the perturbations of specific wave numbers $\kappa$. In this region, stationary patterns can be observed. To see the effect of parameter $\delta$ well, we plot in Figure 2 the dispersion relation corresponding to several values of $\delta$ while keeping the other parameters fixed. We see that the available Turing modes shift to higher wave numbers when $\beta$ decreases.

\section{Spatial Dynamics of Systems (4a) and (4b)}

In the following, we use multiple scale analysis to determine the amplitude equations when $|\kappa|=\kappa_{T}$. Denote $\delta$ as the controlled parameters. When the controlled parameter is larger than the critical value of Turing point, the solutions of systems (4a) and (4b) can be expanded as

$$
c=c_{0}+\sum_{i=1}^{N}\left(A_{i} \exp \left(i \kappa_{i} \vec{r}\right)+\left(\bar{A}_{i} \exp \left(-i \kappa_{i} \vec{r}\right)\right)\right.
$$

with $|\kappa|=\kappa_{T} . A_{j}$ and the conjugate $\bar{A}_{j}$ are the amplitudes associated with the modes $\kappa_{j}$ and $-\kappa_{j}$.

Close to onset $\beta=\beta_{T}$, one has that

$$
\frac{\partial A_{i}}{\partial t}=s_{i} A_{i}+F_{i}\left(A_{i}, A_{j}, \ldots\right) .
$$

Based on the center manifold near the Turing bifurcation point, it can be concluded that amplitude $A_{j}$ satisfies

$$
\frac{\partial A_{i}}{\partial t}=F_{i}\left(A_{i}, \bar{A}_{i}, A_{j}, \bar{A}_{j}, \ldots\right) .
$$




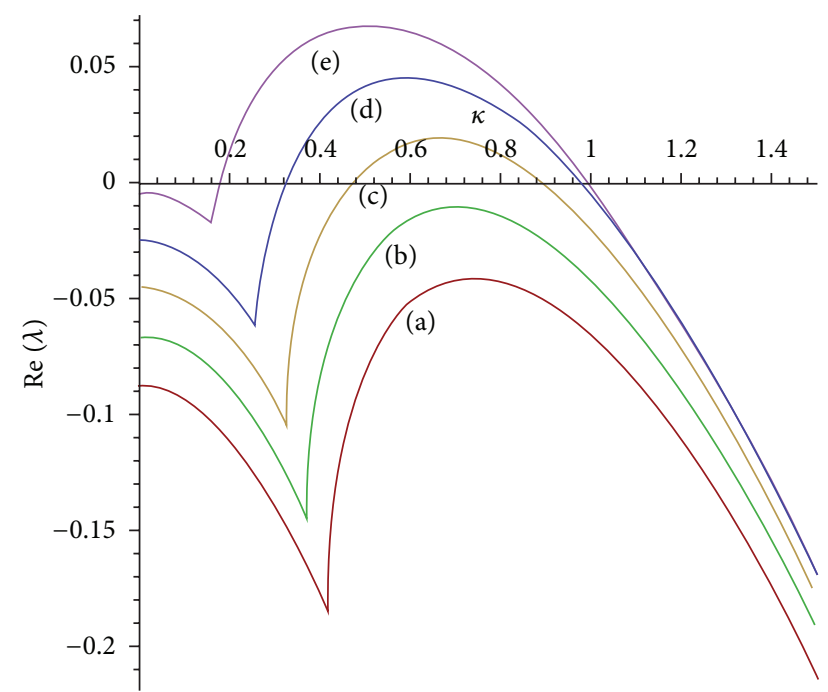

FIGURE 2: Dispersion relation for different $\delta$. Parameters values: $\beta=$ $0.02, \gamma=1.5$, and $\varepsilon=0.15$. (a) $\delta=1.08$; (b) $\delta=1.04$; (c) $\delta=1$; (d) $\delta=0.96$; and (e) $\delta=0.92$.

In order to obtain the amplitude equations, we first need to investigate the linearized form of systems (4a) and (4b) at the equilibrium point $E_{4}$. By setting $u=u^{*}+x$ and $v=v^{*}+y$, we have the following equations:

$$
\begin{gathered}
\frac{\partial x}{\partial t}=\left[2 \gamma u^{*}-3 \gamma\left(u^{*}\right)^{2}+2 \gamma\left(u^{*}\right)^{2} \beta-\gamma \beta-v^{*}\right] x \\
+\left(\beta \gamma-3 \gamma u^{*}+\gamma\right) x^{2}-\gamma x^{3}-x y+\Delta x, \\
\frac{\partial y}{\partial t}=v^{*} x+u^{*} y+x y-2 \delta v^{*} y-\delta y^{2}+\varepsilon \Delta y .
\end{gathered}
$$

Close to onset $\delta=\delta_{T}$, the solutions of systems (4a) and (4b) can be expanded as series form:

$$
U=U_{s}+\sum_{j=1}^{3} U_{0}\left[A_{j} \exp \left(i \kappa_{j} \vec{r}\right)+\bar{A}_{j} \exp \left(-i \kappa_{j} \vec{r}\right)\right]
$$

System (19) can be expanded as

$$
U^{*}=\sum_{j=1}^{3} U_{0}\left[A_{j} \exp \left(i \kappa_{j} \vec{r}\right)+\bar{A}_{j} \exp \left(-i \kappa_{j} \vec{r}\right)\right]
$$

where $U_{0}=\left(\left(a_{11}^{*} \varepsilon+a_{11}^{*}\right) /\left(2 a_{21}^{*}\right), 1\right)^{T}$ is the eigenvector of the linearized operator.

From the standard multiple scale analysis, up to the third order in the perturbations, the spatiotemporal evolution of the amplitudes can be described as

$$
\tau \frac{\partial A_{k}}{\partial t}=\mu A_{k}+\sum_{l m} h_{l m} A_{l} A_{m}+\sum_{l m n} g_{l m n} A_{l} A_{m} A_{n} .
$$

Due to spatial translational symmetry, we have the following equation:

$$
\begin{aligned}
\tau \frac{\partial A_{k}}{\partial t} \exp \left(i \kappa_{k} r_{0}\right) \\
=\mu A_{k} \exp \left(i \kappa_{k} r_{0}\right)+\sum_{l m} h_{l m} A_{l} A_{m} \exp \left[i\left(\kappa_{l}+\kappa_{m}\right) r_{0}\right] \\
\quad+\sum_{l m n} g_{l m n} A_{l} A_{m} A_{n} \exp \left[i\left(\kappa_{l}+\kappa_{m}+\kappa_{n}\right) r_{0}\right] .
\end{aligned}
$$

Comparing (25) with (26), one can find that the two equations hold only if $\kappa_{k}=\kappa_{l}+\cdots+\kappa_{m}$. From the center manifold theory, we know that amplitude equations do not include the amplitude with unstable mode. As a result, we have the following equations:

$$
\begin{aligned}
\tau_{0} \frac{\partial A_{1}}{d t}= & \mu A_{1}+h \bar{A}_{2} \bar{A}_{3} \\
& -\left(g_{1}\left|A_{1}\right|^{2}+g_{2}\left(\left|A_{2}\right|^{2}+\left|A_{3}\right|^{2}\right)\right) A_{1}, \\
\tau_{0} \frac{\partial A_{2}}{d t}= & \mu A_{2}+h \bar{A}_{1} \bar{A}_{3} \\
& -\left(g_{1}\left|A_{2}\right|^{2}+g_{2}\left(\left|A_{1}\right|^{2}+\left|A_{3}\right|^{2}\right)\right) A_{2}, \\
\tau_{0} \frac{\partial A_{3}}{d t}= & \mu A_{3}+h \bar{A}_{1} \bar{A}_{2} \\
& -\left(g_{1}\left|A_{3}\right|^{2}+g_{2}\left(\left|A_{1}\right|^{2}+\left|A_{2}\right|^{2}\right)\right) A_{3},
\end{aligned}
$$

where $\mu=\left(\delta_{T}-\delta\right) / \delta_{T}$ and $\tau_{0}$ is a typical relaxation time.

In the following part, we will give the expressions of $\tau_{0}, h$, $g_{1}$, and $g_{2}$. Let

$$
\begin{aligned}
X & =\left(\begin{array}{l}
x \\
y
\end{array}\right), \\
N & =\left(\begin{array}{l}
N_{1} \\
N_{2}
\end{array}\right) .
\end{aligned}
$$

Then systems (4a) and (4b) can be written as:

$$
\frac{\partial X}{\partial t}=L X+N
$$

where

$$
\begin{gathered}
L=\left(\begin{array}{cc}
2 \gamma u^{*}-3 \gamma\left(u^{*}\right)^{2}+2 \gamma \beta\left(u^{*}\right)^{2}-\gamma \beta-v^{*}+\Delta & 0 \\
v^{*} & u^{*}-2 \delta v^{*}+\varepsilon \Delta
\end{array}\right), \\
N=\left(\begin{array}{c}
\left(\beta \gamma-3 \gamma u^{*}+\gamma\right)-\gamma x^{3}-x y \\
x y-\delta y^{2}
\end{array}\right) .
\end{gathered}
$$

We need to investigate the dynamical behavior when $\delta$ is close to $\delta_{T}$, and thus we expand $\delta$ as:

$$
\delta_{T}-\delta=\epsilon \delta_{1}+\epsilon^{2} \delta_{2}+\epsilon^{3} \delta_{3}+O\left(\epsilon^{4}\right),
$$


where $\epsilon$ is a small enough parameter. We expand $X$ and $N$ as the series form of $\epsilon$ :

$$
\begin{gathered}
X=\left(\begin{array}{l}
x \\
y
\end{array}\right)=\epsilon\left(\begin{array}{l}
x_{1} \\
y_{1}
\end{array}\right)+\epsilon^{2}\left(\begin{array}{l}
x_{2} \\
y_{2}
\end{array}\right)+\epsilon^{3}\left(\begin{array}{l}
x_{3} \\
y_{3}
\end{array}\right)+\cdots, \\
N=\left(\begin{array}{c}
\left(\beta \gamma-3 \gamma u^{*}+\gamma\right)\left(x_{1}^{2} \epsilon^{2}+2 x_{1} x_{2} \epsilon^{3}\right)-\gamma x_{1}^{3} \epsilon^{3}-x_{1} y_{1} \epsilon^{2}-\left(x_{2} y_{1}+x_{1} y_{2}\right) \epsilon^{3}+o\left(\epsilon^{4}\right) \\
x_{1} y_{1} \epsilon^{2}+\left(x_{2} y_{1}+x_{1} y_{2}\right) \epsilon^{3}+o\left(\epsilon^{4}\right)
\end{array}\right) .
\end{gathered}
$$

Linear operator $L$ can be expanded as

$$
L=L_{T}+\left(\delta_{T}-\delta\right) M,
$$

where

$$
L_{T}=\left(\begin{array}{cc}
a_{11}^{*}+\Delta & a_{12}^{*} \\
a_{21}^{*} & a_{22}^{*}+\varepsilon \Delta
\end{array}\right), \quad M=\left(\begin{array}{ll}
b_{11} & b_{12} \\
b_{21} & b_{22}
\end{array}\right) .
$$

Let

$$
T_{0}=t, \quad T_{1}=\epsilon t, \quad T_{2}=\epsilon^{2} t,
$$

and $T_{i}$ is a dependent variable. For the derivation of time, we have that

$$
\frac{\partial}{\partial t}=\frac{\partial}{\partial T_{0}}+\epsilon \frac{\partial}{\partial T_{1}}+\epsilon^{2} \frac{\partial}{\partial T_{2}}+o\left(\epsilon^{3}\right) .
$$
form:

The solutions of systems (4a) and (4b) have the following

$$
X=\left(\begin{array}{l}
x \\
y
\end{array}\right)=\sum_{i=1}^{3}\left(\begin{array}{c}
A_{i}^{x} \\
A_{i}^{y}
\end{array}\right) \exp \left(i \kappa_{i} \vec{r}\right)+\cdots .
$$

This expression implies that the bases of the solutions have nothing to do with time and the amplitude $A$ is a variable that changes slowly. As a result, one has the following equation:

$$
\frac{\partial A}{\partial t}=\epsilon \frac{\partial A}{\partial T_{1}}+\epsilon^{2} \frac{\partial A}{\partial T_{2}}+o\left(\epsilon^{3}\right) .
$$

Substituting the above equations into (29) and expanding (29) according to different orders of $\epsilon$, we can obtain three equations as follows:

$$
\begin{aligned}
& \epsilon: L_{T}\left(\begin{array}{l}
x_{1} \\
y_{1}
\end{array}\right)=0 \\
& \epsilon^{2}: L_{T}\left(\begin{array}{l}
x_{2} \\
y_{2}
\end{array}\right)= \frac{\partial}{\partial T_{1}}\left(\begin{array}{l}
x_{1} \\
y_{1}
\end{array}\right)-\delta_{1} M\left(\begin{array}{l}
x_{1} \\
y_{1}
\end{array}\right) \\
&-\left(\begin{array}{r}
\left(\beta \gamma-3 \gamma u^{*}+\gamma\right) x_{1}^{2}-x_{1} y_{1} \\
x_{1} y_{1}
\end{array}\right) ; \\
& \epsilon^{3}: L_{T}\left(\begin{array}{l}
x_{3} \\
y_{3}
\end{array}\right)= \frac{\partial}{\partial T_{1}}\left(\begin{array}{l}
x_{2} \\
y_{2}
\end{array}\right)+\frac{\partial}{\partial T_{2}}\left(\begin{array}{l}
x_{1} \\
y_{1}
\end{array}\right)-\delta_{1} M\left(\begin{array}{l}
x_{2} \\
y_{2}
\end{array}\right) \\
&-\delta_{2} M\left(\begin{array}{l}
x_{1} \\
y_{1}
\end{array}\right)-E
\end{aligned}
$$

where

$$
E=\left(\begin{array}{c}
2 x_{1} x_{2}\left(\beta \gamma-3 \gamma u^{*}+\gamma\right)-\gamma x_{1}^{3}-\left(x_{2} y_{1}+x_{1} y_{2}\right) \\
x_{2} y_{1}+x_{1} y_{2}
\end{array}\right)
$$

We first consider the case of the first order of $\varepsilon$. Since $L_{T}$ is the linear operator of the system close to the onset, $\left(x_{1}, y_{1}\right)^{T}$ is the linear combination of the eigenvectors that corresponds to the eigenvalue zero. Since that

$$
\left(\begin{array}{l}
x \\
y
\end{array}\right)=\sum_{i=1}^{3}\left(\begin{array}{c}
A_{i}^{x} \\
A_{i}^{y}
\end{array}\right) \exp \left(i \kappa_{i} \vec{r}\right)+\text { c.c. },
$$

we have that

$$
\begin{aligned}
& \left(a_{11}^{*}+\Delta\right) x_{1}+a_{12}^{*} y_{1}=0 \\
& a_{21}^{*} x_{1}+\left(a_{22}^{*}+\varepsilon \Delta\right) y_{1}=0 .
\end{aligned}
$$

As $\varepsilon a_{12}^{*}=\left(\left(a_{22}^{*}-\varepsilon a_{11}^{*}\right) / 2 a_{21}^{*}\right)^{2}$, we can obtain that $x_{1}=\left(a_{22}^{*}-\right.$ $\left.\varepsilon a_{11}^{*}\right) /\left(2 a_{21}^{*}\right)$ by assuming $y_{1}=1$.

Let $R=\left(a_{11}^{*} \varepsilon-a_{22}^{*}\right) / 2 a_{21}^{*}$ then

$$
\begin{gathered}
\left(\begin{array}{c}
x_{1} \\
y_{1}
\end{array}\right)=\left(\begin{array}{c}
R \\
1
\end{array}\right)\left(W_{1} \exp \left(i \kappa_{1} \vec{r}\right)+W_{2} \exp \left(i \kappa_{2} \vec{r}\right)\right. \\
\left.+W_{3} \exp \left(i \kappa_{3} \vec{r}\right)\right)+ \text { c.c. }
\end{gathered}
$$

where $\left|\kappa_{j}\right|=\kappa_{T}^{*}$ and $W_{j}$ is the amplitude of the mode $\exp \left(i \kappa_{j} r\right)$.

Now, we consider the case of the second order of $\varepsilon$. Note that

$$
\begin{aligned}
L_{T}\left(\begin{array}{l}
x_{2} \\
y_{2}
\end{array}\right)= & \frac{\partial}{\partial T_{1}}\left(\begin{array}{l}
x_{1} \\
y_{1}
\end{array}\right)-\delta_{T}\left(\begin{array}{l}
b_{11} x_{1}+b_{12} y_{1} \\
b_{21} x_{1}+b_{22} y_{1}
\end{array}\right) \\
& -\left(\begin{array}{c}
\left(\beta \gamma-3 \gamma u^{*}+\gamma\right) x_{1}^{2}-x_{1} y_{1} \\
x_{1} y_{1}
\end{array}\right) \\
= & \left(\begin{array}{l}
F_{x} \\
F_{y}
\end{array}\right) .
\end{aligned}
$$

According to the Fredholm solubility condition, the vector function of the right hand of the above equation must be orthogonal with the zero eigenvectors of operator $\mathbf{L}_{c}^{+}$. And the zero eigenvectors of operator $\mathbf{L}_{c}^{+}$are

$$
\left(\begin{array}{c}
1 \\
-\frac{1}{\varepsilon} R
\end{array}\right) \exp \left(i \kappa_{j} \vec{r}\right)+c . c \quad(j=1,2,3)
$$

It can be found from the orthogonality condition that

$$
\left(\begin{array}{c}
1 \\
-\frac{1}{\varepsilon} R
\end{array}\right)\left(\begin{array}{c}
F_{x}^{i} \\
F_{y}^{i}
\end{array}\right)=0
$$

where $F_{x}^{i}$ and $F_{y}^{i}$ represent the coefficients corresponding to $\exp \left(i \kappa_{j} r\right)$ in $F_{x}$ and $F_{y}$. 
By investigating $\exp \left(i \kappa_{1} \vec{r}\right)$, one has

$$
\begin{aligned}
\left(\begin{array}{c}
F_{x}^{1} \\
F_{y}^{1}
\end{array}\right)= & \left(\begin{array}{c}
R \frac{\partial W_{1}}{\partial T_{1}} \\
\frac{\partial W_{1}}{\partial T_{1}}
\end{array}\right)-\delta_{1}\left(\begin{array}{c}
b_{11} R W_{1}+b_{12} W_{1} \\
b_{21} R W_{1}+b_{22} W_{1}
\end{array}\right) \\
& -\left(\begin{array}{c}
2 R^{2}\left(\beta \gamma-3 \gamma u^{*}+\gamma\right) \bar{W}_{2} \bar{W}_{3}+2 R \bar{W}_{2} \bar{W}_{3} \\
2 R \bar{W}_{2} \bar{W}_{3}
\end{array}\right) .
\end{aligned}
$$

It can be obtained from the orthogonality condition that

$$
\begin{aligned}
\frac{\varepsilon-1}{\varepsilon} R \frac{\partial W_{1}}{\partial T_{1}}= & \delta\left(R b_{11}+b_{22}-\frac{R}{\varepsilon}\left(R b_{21}+b_{22}\right) W_{1}\right) \\
& +2 R^{2}\left(\beta \gamma-3 \gamma u^{*}+\gamma+\frac{1}{R}-\frac{1}{\varepsilon}\right) \bar{W}_{2} \bar{W}_{3}
\end{aligned}
$$

By using the same methods, one has

$$
\begin{aligned}
\left(\begin{array}{l}
x_{2} \\
y_{2}
\end{array}\right)= & \left(\begin{array}{l}
X_{0} \\
Y_{0}
\end{array}\right)+\sum_{j=1}^{3}\left(\begin{array}{l}
X_{j} \\
Y_{j}
\end{array}\right) \exp \left(i \kappa_{j} \vec{r}\right) \\
& +\sum_{j=1}^{3}\left(\begin{array}{c}
X_{j j} \\
Y_{j j}
\end{array}\right) \exp \left(2 i \kappa_{j} \vec{r}\right)+Q+\text { c.c. },
\end{aligned}
$$

where

$$
\begin{aligned}
Q= & \left(\begin{array}{c}
X_{12} \\
Y_{12}
\end{array}\right) \exp \left(i\left(\kappa_{1}-\kappa_{2}\right) \vec{r}\right)+\left(\begin{array}{c}
X_{23} \\
Y_{23}
\end{array}\right) \exp \left(i\left(\kappa_{2}-\kappa_{3}\right) \vec{r}\right) \\
& +\left(\begin{array}{c}
X_{31} \\
Y_{31}
\end{array}\right) \exp \left(i\left(\kappa_{3}-\kappa_{1}\right) \vec{r}\right)
\end{aligned}
$$

By solving the sets of the linear equations about $\exp (0)$, $\exp \left(i \kappa_{j} \vec{r}\right), \exp \left(2 i \kappa_{j} \vec{r}\right)$, and $\exp \left(i\left(\kappa_{j}-\kappa_{k}\right) \vec{r}\right)$, we obtain that

$$
\begin{aligned}
\left(\begin{array}{c}
X_{0} \\
Y_{0}
\end{array}\right) & \\
= & \left(\begin{array}{c}
\frac{a_{22}^{*}\left[-2 R^{2}\left(\beta \gamma-3 \gamma u^{*}+\gamma\right)+2 R\right]+2 R a_{12}^{*}}{a_{11}^{*} a_{22}^{*}-a_{12}^{*} a_{21}^{*}} \\
\frac{a_{21}^{*}\left[2 R^{2}\left(\beta \gamma-3 \gamma u^{*}+\gamma\right)-2 R\right]+2 R a_{11}^{*}}{a_{11}^{*} a_{22}^{*}-a_{12}^{*} a_{21}^{*}}
\end{array}\right) \\
& \times\left(\left|W_{1}\right|^{2}+\left|W_{2}\right|^{2}+\left|W_{3}\right|^{2}\right)
\end{aligned}
$$

$$
\begin{aligned}
& \left(\begin{array}{l}
X_{j j} \\
Y_{j j}
\end{array}\right) \\
& =\left(\begin{array}{l}
\frac{\left(a_{22}^{*}-4 \varepsilon \kappa_{T}^{2}\right)\left[-R^{2}\left(\beta \gamma-3 \gamma u^{*}+\gamma\right)+R\right]+R a_{12}^{*}}{\left(a_{11}^{*}-4 \kappa_{T}^{2}\right)\left(a_{22}^{*}-4 \varepsilon \kappa_{T}^{2}\right)-a_{12}^{*} a_{21}^{*}} \\
\frac{a_{21}^{*}\left[R^{2}\left(\beta \gamma-3 \gamma u^{*}+\gamma\right)-2 R\right]-R\left(a_{11}^{*}-4 \varepsilon \kappa_{T}^{2}\right)}{\left(a_{11}^{*}-4 \kappa_{T}^{2}\right)\left(a_{22}^{*}-4 \varepsilon \kappa_{T}^{2}\right)-a_{12}^{*} a_{21}^{*}}
\end{array}\right) \\
& \quad \times W_{j}^{2}, \\
& \left(\begin{array}{l}
X_{j k} \\
Y_{j k}
\end{array}\right)
\end{aligned}
$$

$$
\begin{aligned}
& =\left(\begin{array}{c}
\frac{\left(a_{22}^{*}-3 \varepsilon \kappa_{T}^{2}\right)\left[-2 R^{2}\left(\beta \gamma-3 \gamma u^{*}+\gamma\right)+2 R\right]+2 R a_{12}^{*}}{\left(a_{11}^{*}-3 \kappa_{T}^{2}\right)\left(a_{22}^{*}-3 \varepsilon \kappa_{T}^{2}\right)-a_{12}^{*} a_{21}^{*}} \\
\frac{a_{21}^{*}\left[2 R^{2}\left(\beta \gamma-3 \gamma u^{*}+\gamma\right)-2 R\right]-2 R\left(a_{11}^{*}-3 \kappa_{T}^{2}\right)}{\left(a_{11}^{*}-3 \kappa_{T}^{2}\right)\left(a_{22}^{*}-3 \varepsilon \kappa_{T}^{2}\right)-a_{12}^{*} a_{21}^{*}}
\end{array}\right) \\
& \quad \times W_{j} \bar{W}_{k},
\end{aligned}
$$

where $\kappa_{T}^{2}=\sqrt{\left(a_{11}^{*} a_{22}^{*}-a_{12}^{*} a_{21}^{*}\right) / \varepsilon}$.

For the third order of $\varepsilon$, we have that

$$
\begin{aligned}
L_{T}\left(\begin{array}{l}
x_{3} \\
y_{3}
\end{array}\right)= & \frac{\partial}{\partial T_{1}}\left(\begin{array}{l}
x_{2} \\
y_{2}
\end{array}\right)+\frac{\partial}{\partial T_{2}}\left(\begin{array}{l}
x_{1} \\
y_{1}
\end{array}\right) \\
& -\delta_{1} M\left(\begin{array}{l}
x_{2} \\
y_{2}
\end{array}\right)-\delta_{2} M\left(\begin{array}{l}
x_{1} \\
y_{1}
\end{array}\right)-S,
\end{aligned}
$$

where

$$
S=\left(\begin{array}{c}
2 x_{1} x_{2}\left(\beta \gamma-3 \gamma u^{*}\right)-\gamma x_{1}^{3}-\left(x_{2} y_{1}+x_{1} y_{2}\right) \\
x_{2} y_{1}+x_{1} y_{2}
\end{array}\right)
$$

Using the Fredholm solubility condition, we can obtain

$$
\begin{aligned}
\frac{\varepsilon-1}{\varepsilon} R & \frac{\partial W_{1}}{\partial T_{2}}+\frac{\varepsilon-1}{\varepsilon} R \frac{\partial Y_{1}}{\partial T_{1}} \\
= & \delta_{2}\left[R b_{11}+b_{12}-\frac{1}{\varepsilon} R\left(R b_{21}+b_{22}\right)\right] W_{1} \\
& +\delta_{1}\left[R b_{11}+b_{12}-\frac{1}{\varepsilon}\left(R b_{21}+b_{22}\right)\right] Y_{1}+Z
\end{aligned}
$$

where

$$
\begin{aligned}
Z= & {\left[2 R^{2}\left(\beta \gamma-3 \gamma u^{*}+\gamma\right)-2 R-2 \frac{1}{\varepsilon}\right] } \\
& \times\left[W_{1} Y_{0}+W-2 Y_{12}+W_{3} Y_{13}+\bar{W}_{1} Y_{11}\right. \\
& \left.+\bar{W}_{2} \bar{Y}_{3}+\bar{W}_{3} \bar{Y}_{2}\right] \\
& -\left(G_{1}\left|W_{1}\right|^{2}+G_{2}\left|W_{2}\right|^{2}+G_{3}\left|W_{3}\right|^{2}\right) W_{1},
\end{aligned}
$$


TABLE 1: Coefficients for different parameter sets.

\begin{tabular}{ccccccccc}
\hline$\beta$ & $\delta$ & $h$ & $g_{1}$ & $g_{2}$ & $\mu_{1}$ & $\mu_{2}$ & $\mu_{3}$ \\
\hline 0.02 & 0.92 & -19.08604 & 7599.215 & 6906.578 & 0.0042531 & 0 & 5.770186 \\
0.02 & 0.96 & 2.1329690 & -740.11 & -1429.72 & -0.000315 & 0 & -0.00708 & -0.00611 \\
0.02 & 1 & 8.4304106 & -207.521 & -474.186 & -0.015371 & 0 & -0.20741 & -0.01250 \\
0.02 & 1.12 & 11.304093 & -99.3194 & -193.856 & -0.0655924 & 0 & -1.42005 & -0.04391 \\
\hline
\end{tabular}

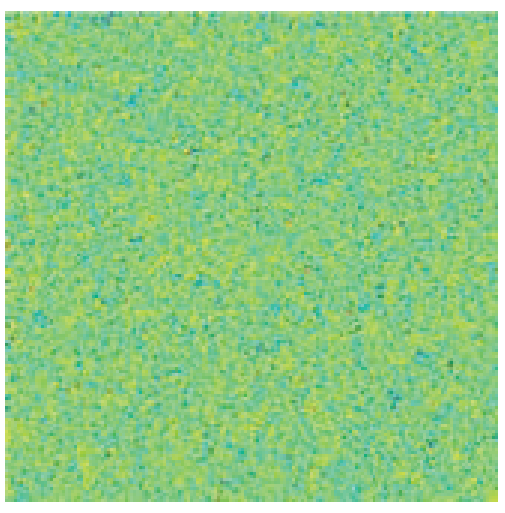

(a)

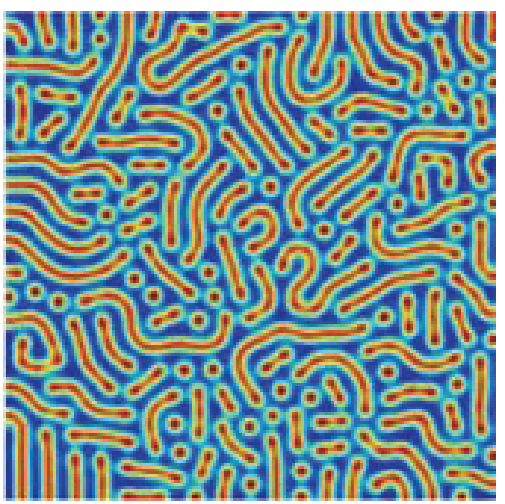

(d)

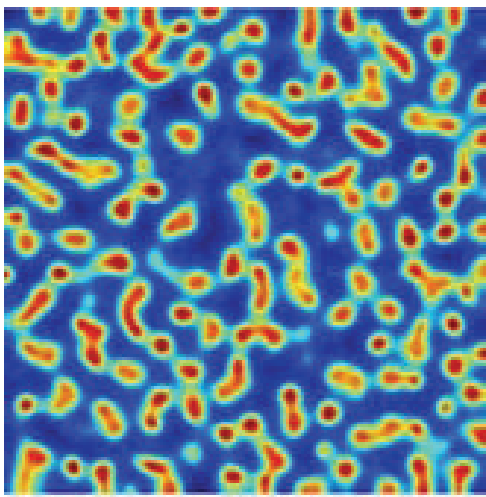

(b)

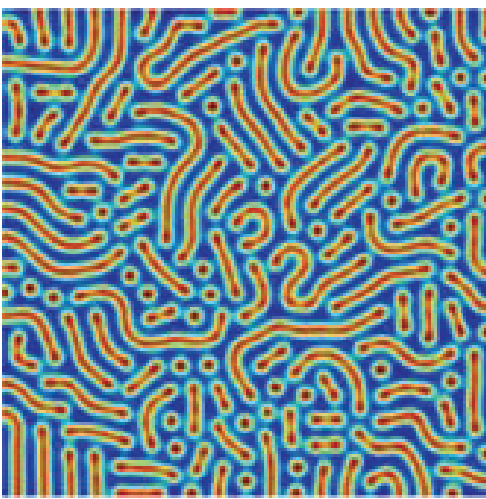

(e)

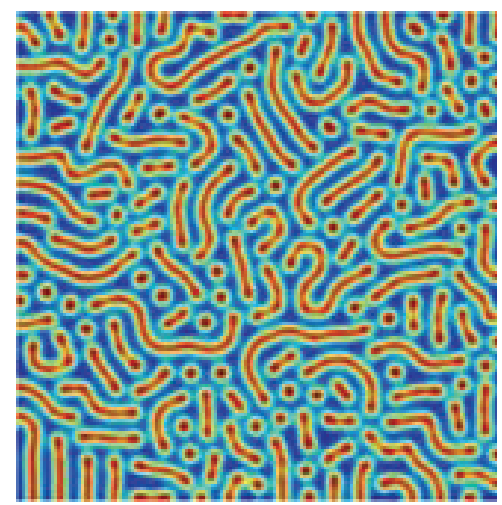

(c)

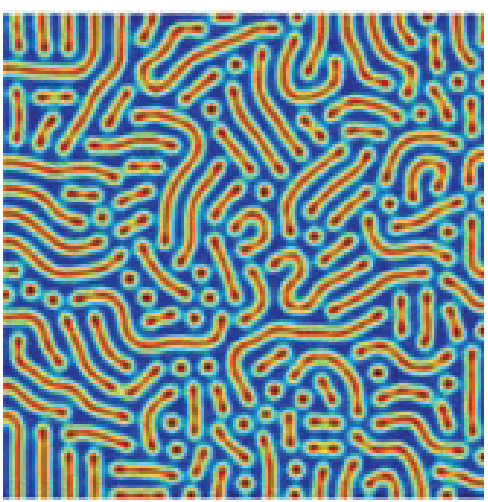

(f)

Figure 3: Spatial pattern of prey population at different time. Parameters set: $\gamma=1.5, \varepsilon=0.15$, and $\delta=0.92$. (a) $t=0$; (b) $t=100$; (c) $t=200$; (d) $t=500$; (e) $t=1000$; and (f) $t=2000$.

$$
\begin{aligned}
G_{1}= & \left(\frac{1}{\varepsilon} R-1\right)\left[R\left(y_{11}+y_{0}\right)+x_{11}+x_{0}\right] \\
& -2 R\left(x_{11}+x_{0}\right)\left(\beta \gamma-3 \gamma u^{*}+\gamma\right)+3 \gamma R^{3}, \\
G_{2}= & \left(\frac{1}{\varepsilon} R-1\right)\left[R\left(y_{12}+y_{0}\right)+x_{12}+x_{0}\right] \\
& -2 R\left(x_{12}+x_{0}\right)\left(\beta \gamma-3 \gamma u^{*}+\gamma\right)+6 \gamma R^{3} .
\end{aligned}
$$

By using the same methods, we can obtain the other two equations. The amplitude $A_{i}$ can be expanded as

$$
A_{i}=\epsilon W_{i}+\epsilon^{2} V_{i}+o\left(\epsilon^{3}\right) \text {. }
$$

As a result, we have

$$
\tau_{0} \frac{\partial A_{1}}{\partial t}=\mu A_{1}+h \bar{A}_{2} \bar{A}_{3}-\left(g_{1}\left|A_{1}\right|^{2}+g_{2}\left|A_{2}\right|^{2}+\left|A_{3}\right|^{2}\right) A_{1} \text {. }
$$

The other two equations can be obtained through the transformation of the subscript of $A$. By calculations, we obtain the expressions of the coefficients of $\tau_{0}, h, g_{1}$, and $g_{2}$ as follows:

$$
\begin{gathered}
\tau_{0}=R \frac{\varepsilon-1}{\delta_{T}\left[R b_{11}+b_{12}-(R / \varepsilon)\left(R b_{21}+b_{22}\right)\right]}, \\
h=\frac{\left[2 R^{2}\left(\beta \gamma-3 \gamma u^{*}+\gamma\right)-2 R-2\left(R^{2} / \varepsilon\right)\right]}{\delta_{T}\left[R b_{11}+b_{12}-(R / \varepsilon)\left(R b_{21}+b_{22}\right)\right]} \\
g_{1}=\frac{G_{1}}{\delta_{T}\left[R b_{11}+b_{12}-(R / \varepsilon)\left(R b_{21}+b_{22}\right)\right]} \\
g_{2}=\frac{G_{2}}{\delta_{T}\left[R b_{11}+b_{12}-(R / \varepsilon)\left(R b_{21}+b_{22}\right)\right]}
\end{gathered}
$$




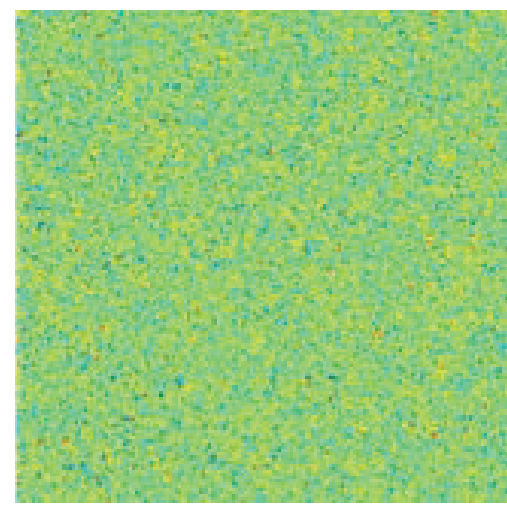

(a)

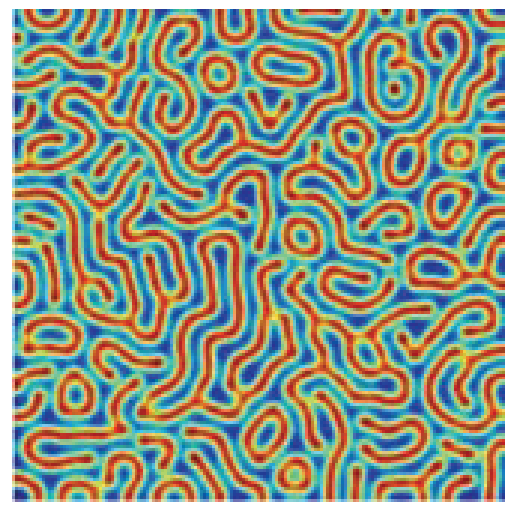

(d)

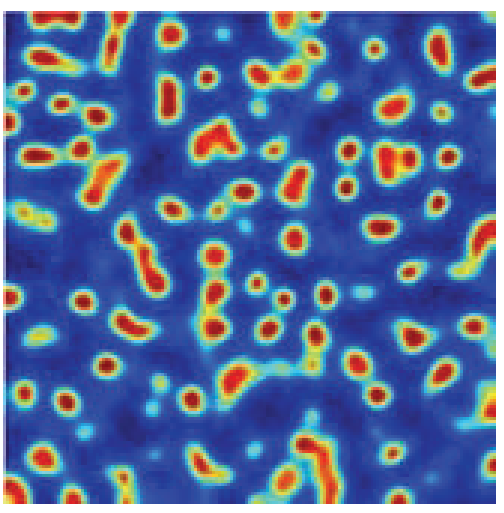

(b)

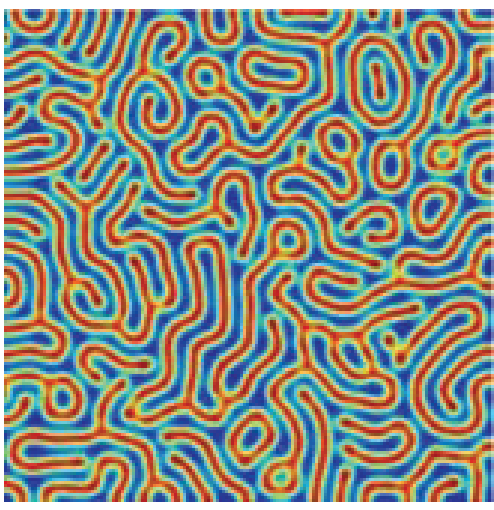

(e)

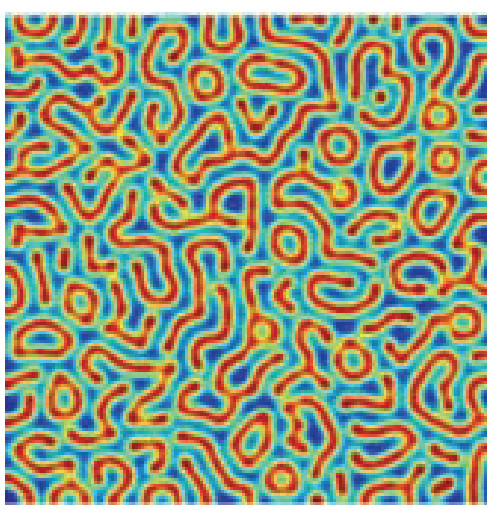

(c)

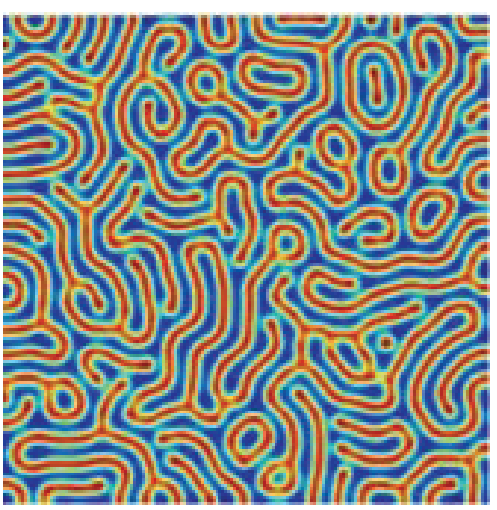

(f)

Figure 4: Spatial pattern of prey population at different time. Parameters set: $\gamma=1.5, \varepsilon=0.15$, and $\delta=0.96$. (a) $t=0$; (b) $t=50$; (c) $t=100$; (d) $t=200$; (e) $t=500$; and (f) $t=1000$.

where $G_{1}=((R / \varepsilon)-1)\left[R\left(y_{0}+y_{11}\right)+x_{0}+x_{11}\right]-2 R\left(x_{0}+\right.$ $\left.x_{11}\right)\left(\beta \gamma-3 \gamma u^{*}+\gamma\right)+3 \gamma R^{3}$ and $G_{2}=((R / \varepsilon)-1)\left[R\left(y_{0}+y_{12}\right)+\right.$ $\left.x_{0}+x_{12}\right]-2 R\left(x_{0}+x_{12}\right)\left(\beta \gamma-3 \gamma u^{*}+\gamma\right)+6 \gamma R^{3}$.

By using substitutions, we have

$$
\begin{gathered}
\tau_{0} \frac{\partial \varphi}{d t}=-h \frac{\rho_{1}^{2} \rho_{2}^{2}+\rho_{1}^{2} \rho_{3}^{2}+\rho_{2}^{2} \rho_{3}^{2}}{\rho_{1} \rho_{2} \rho_{3}} \sin \varphi, \\
\tau_{0} \frac{\partial \rho_{1}}{d t}=\mu \rho_{1}+h \rho_{2} \rho_{3} \cos \varphi-g_{1} \rho_{1}^{3}-g_{2}\left(\rho_{2}^{2} \rho_{3}^{2}\right) \rho_{1}, \\
\tau_{0} \frac{\partial \rho_{2}}{d t}=\mu \rho_{2}+h \rho_{1} \rho_{3} \cos \varphi-g_{1} \rho_{2}^{3}-g_{2}\left(\rho_{1}^{2} \rho_{3}^{2}\right) \rho_{2}, \\
\tau_{0} \frac{\partial \rho_{3}}{d t}=\mu \rho_{3}+h \rho_{1} \rho_{2} \cos \varphi-g_{1} \rho_{3}^{3}-g_{2}\left(\rho_{1}^{2} \rho_{2}^{2}\right) \rho_{3},
\end{gathered}
$$

where $\varphi=\varphi_{1}+\varphi_{2}+\varphi_{3}$. In order to see the relationships between different parameters, we give the values of coefficients for different parameter sets in Table 1.

The dynamical systems (4a) and (4b) possess five kinds of solutions [23] as follows.

(1) The stationary state $(O)$, given by

$$
\rho_{1}=\rho_{2}=\rho_{3}=0 \text {, }
$$

is stable for $\mu<\mu_{2}=0$ and unstable for $\mu>\mu_{2}$.
(2) Stripe patterns $(S)$, given by

$$
\rho_{1}=\sqrt{\frac{\mu}{g_{1}}} \neq 0, \quad \rho_{2}=\rho_{3}=0,
$$

are stable for $\mu>\mu_{3}=h^{2} g_{1} /\left(g_{2}-g_{1}\right)^{2}$, and unstable for $\mu<\mu_{3}$.

(3) Hexagon patterns $\left(H_{0}, H_{\pi}\right)$ are given by

$$
\rho_{1}=\rho_{2}=\rho_{3}=\frac{|h| \pm \sqrt{h^{2}+4\left(g_{1}+2 g_{2} \mu\right)}}{2\left(g_{1}+2 g_{2}\right)},
$$

with $\varphi=0$ or $\pi$, and exist when

$$
\mu>\mu_{1}=\frac{-h^{2}}{4\left(g_{1}+2 g_{2}\right)} .
$$

The solution $\rho^{+}=|h|+\sqrt{h^{2}+4\left(g_{1}+2 g_{2} \mu\right)} / 2\left(g_{1}+\right.$ $\left.2 g_{2}\right)$ is stable only for

$$
\mu<\mu_{4}=\frac{2 g_{1}+g_{2}}{\left(g_{2}-g_{1}\right)^{2}} h^{2},
$$




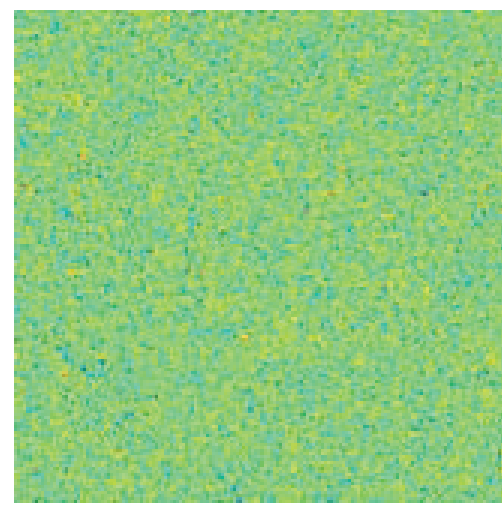

(a)

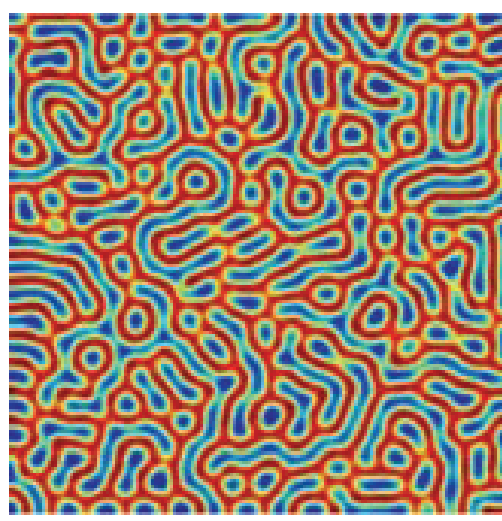

(d)

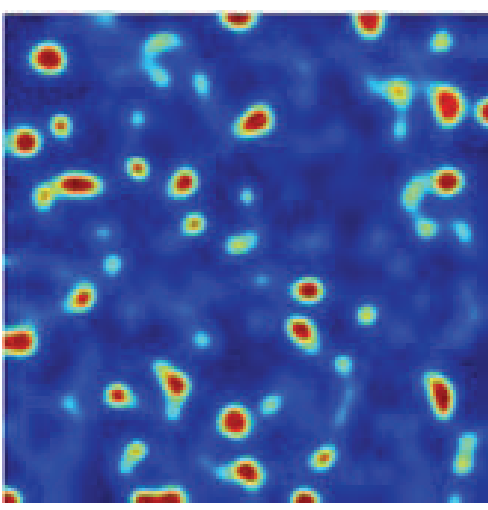

(b)

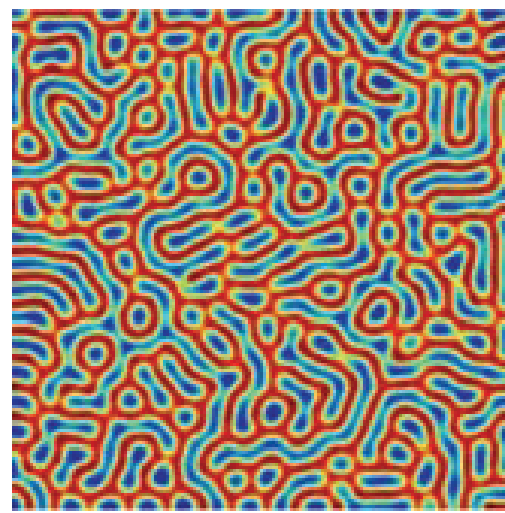

(e)

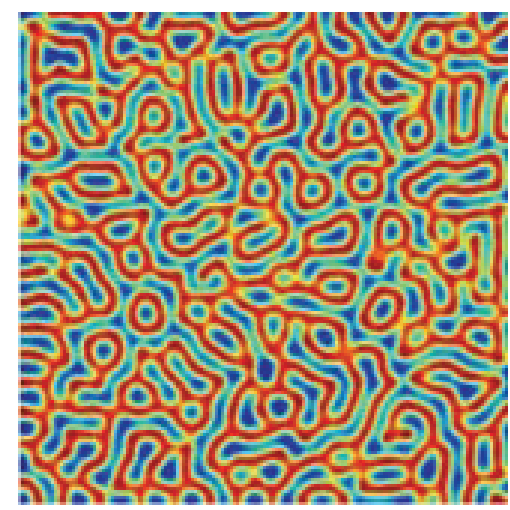

(c)

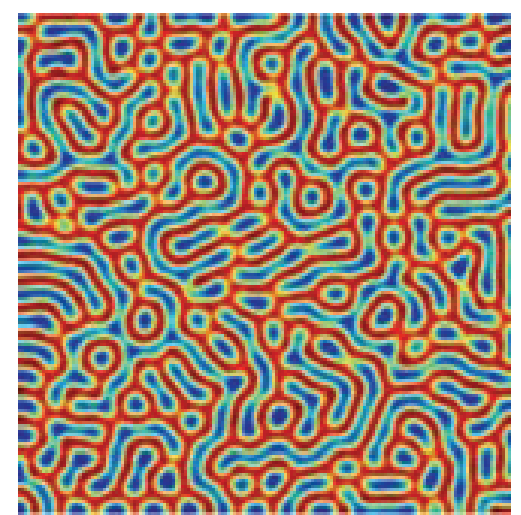

(f)

FIGURE 5: Spatial pattern of prey population at different time. Parameters set: $\gamma=1.5, \varepsilon=0.15$, and $\delta=1$. (a) $t=0$; (b) $t=150$; (c) $t=300$; (d) $t=500$; (e) $t=600$; and (f) $t=1000$.

and $\rho^{-}=\left(|h|-\sqrt{h^{2}+4\left(g_{1}+2 g_{2} \mu\right)}\right) / 2\left(g_{1}+2 g_{2}\right)$ is always unstable.

(4) The mixed states are given by

$$
\rho_{1}=\frac{|h|}{g_{2}-g_{1}}, \quad \rho_{2}=\rho_{3}=\sqrt{\frac{\mu-g_{1} \rho_{1}^{2}}{g_{1}+g_{2}}},
$$

with $g_{2}>g_{1}$. They exist when $\mu>\mu_{3}$ and are always unstable.

\section{Spatial Pattern of Systems (4a) and (4b)}

In this section, we perform extensive numerical simulations of the spatially extended systems (4a) and (4b) in twodimensional spaces. All our numerical simulations employ the zero-flux boundary conditions with a system size of $200 \times$ 200. The space step is $\Delta H=1$, and the time step is $\Delta t=$ 0.00001 .

In Figure 3, we show the spatial pattern of prey population at different time. In the parameter set, $\gamma=1.5, \varepsilon=0.15$, and $\delta=0.92$, we find that $\mu \in\left(\mu_{3}, \mu_{4}\right)$, which means that there is coexistence of spotted and stripe patterns. As shown in this figure, our theoretical results are consistent with the numerical results.
By setting $\gamma=1.5, \varepsilon=0.15$, and $\delta=0.96$, one can obtain that $\mu>\mu_{4}$. In Figure 4 , we show the spatial pattern of prey population when $t$ equals $0,50,100,200,500$, and 1000. At the initial time, the prey population shows patched invasion. As time increases, stripe pattern appears and the structure does not change a lot. While keeping other parameters fixed and increasing $\delta$, we find that stripe pattern will occupy the whole space. However, some stripe patterns connect with each other and cause the emergence of spotted patterns which are shown in Figure 5.

Figure 6 shows the evolution of the spatial pattern of prey population at $t=0,100,300,500,1000$, and 2000 iterations, with small random perturbation of the stationary solution of the spatially homogeneous systems (4a) and (4b). The corresponding parameters values are $\gamma=1.5, \varepsilon=0.15$, and $\delta=1.04$. By the amplitude equations, we can conclude that there are spotted patterns of prey population for this parameter set. In this case, one can see that for the systems (4a) and (4b), the random initial distribution leads to the formation of an irregular transient pattern in the domain. After these forms, it grows slightly and spotted patterns emerge. When the time is large enough, the spotted patterns prevail over the two-dimensional space. As time further increases, the pattern structures of the prey population do not undergo any further changes. 


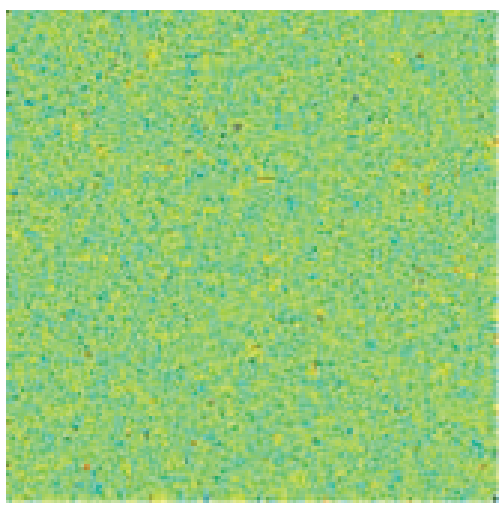

(a)

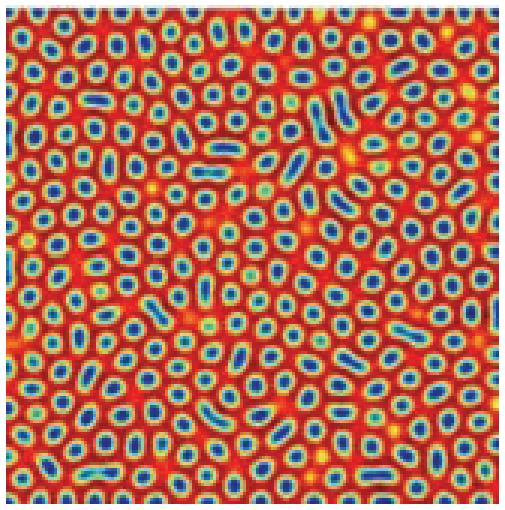

(d)

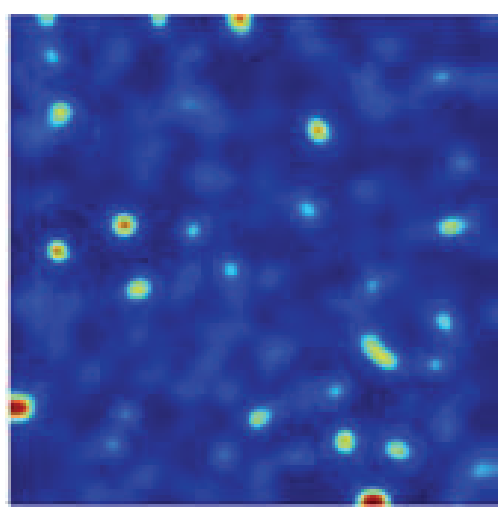

(b)

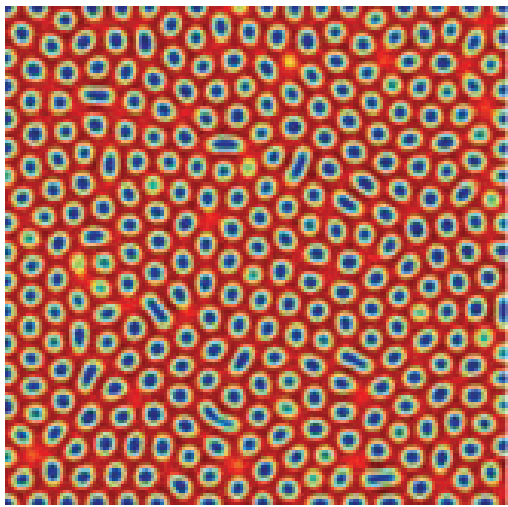

(e)

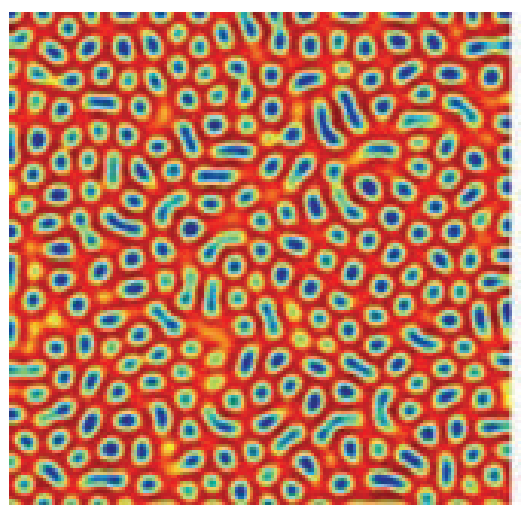

(c)

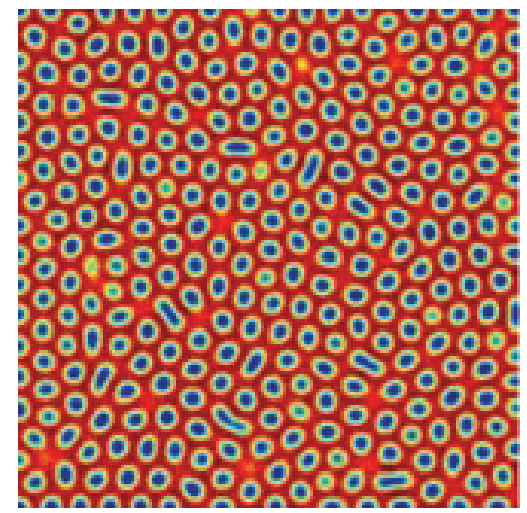

(f)

Figure 6: Spatial pattern of prey population at different time. Parameters set: $\gamma=1.5, \varepsilon=0.15$, and $\delta=1.04$. (a) $t=0$; (b) $t=100$; (c) $t=300$; (d) $t=500$; (e) $t=1000$; and (f) $t=2000$.

\section{Conclusion and Discussion}

Allee effect has been paid much attention due to its strong potential impact on population dynamics [24]. In this paper, we investigated the pattern dynamics of a spatial predatorprey systems with Allee effect. Based on the bifurcation analysis, exact Turing pattern region is obtained. By using amplitude equations, the Turing pattern selection of the predator-prey system is well presented. It is found that the predator-prey systems with Allee effect have rich spatial dynamics by performing a series of numerical simulations.

It should be noted that our results were obtained under the assumption that predation is modeled by the bilinear function of the prey and predator densities. However, this function has limitations to describe many realistic phenomena in the biology. By numerical simulations, we find that the system exhibits similar behaviour when the functional response is of other types, such as Holling-II and Holling-III forms.

To compare the spatial dynamics for different parameters, we give the spatial patterns of population $u$ when the parameter values are out of the domain of Turing space. For this parameter set, systems (4a) and (4b) have Hopf bifurcation, and spiral waves occupy the whole domain instead of stationary patterns, which is shown in Figure 7. The stability of spiral wave can be done by using the spectrum theory analysis $[25,26]$. In the further study, we will use the spectrum theory to show the stability of spiral wave.

In [15], they found that a spatial predator-prey model with Allee effect and linear death rate could increase the system's complexity and enhance chaos in population dynamics. However, in this paper, we showed that a spatial population model with Allee effect and nonlinear death rate can induce stationary patterns, which is different from the previous results.

From a biological point of view, our results show that predator mortality plays an important role in the spatial invasions of populations. More specifically, low predator mortality will induce stationary patterns (cf. Figures 36), and high predator mortality corresponds to travelling patterns (cf. Figure 7). When the populations exhibit wave distribution in space, the dynamics of populations may be accompanied with chaotic properties [27, 28]. If the chaotic behavior occurs, it may lead to the extinction of the population, or the population may be out of control $[29,30]$. In that case, we need to find out the best way to control the chaos or change the chaotic behavior. 


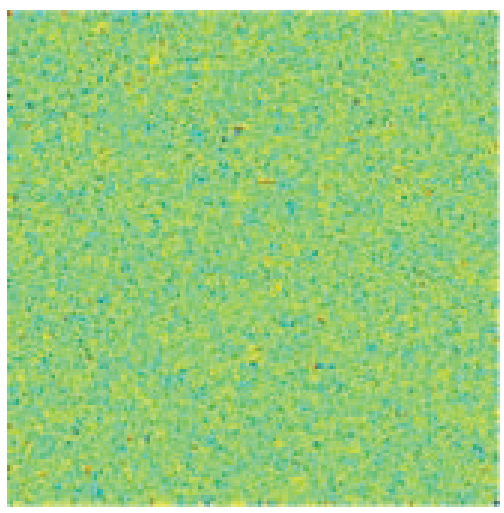

(a)

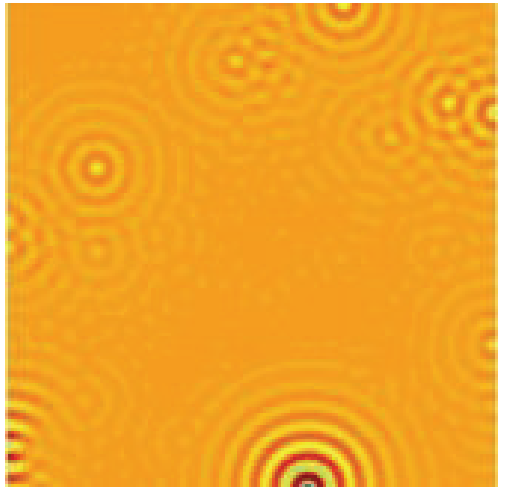

(d)

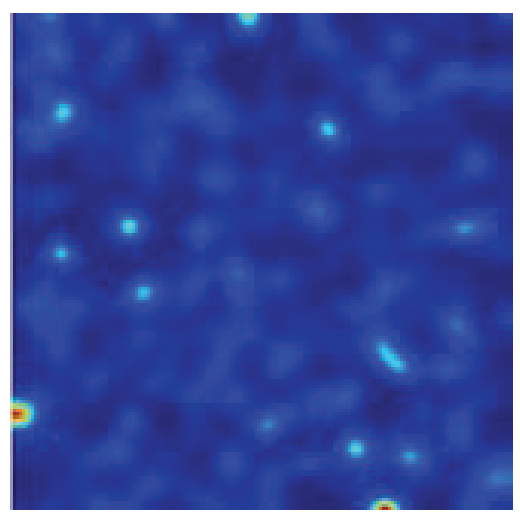

(b)

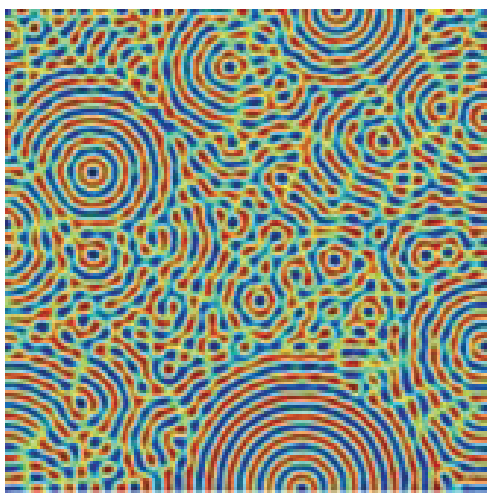

(e)

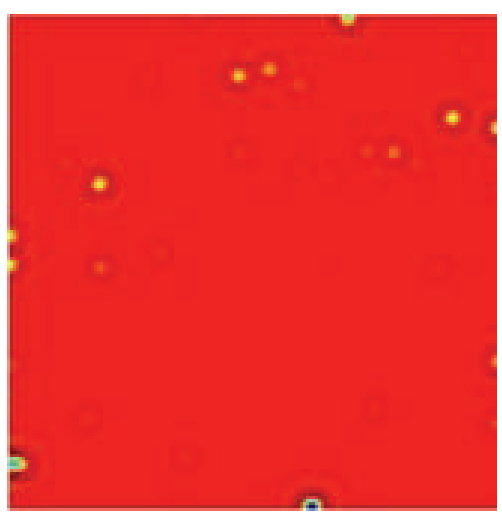

(c)

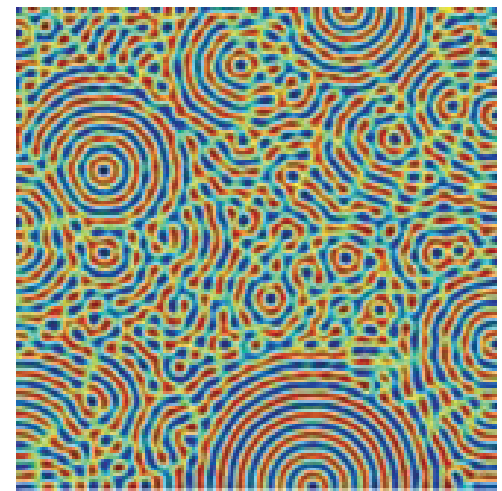

(f)

FIGURE 7: Spatial pattern of prey population at different time. Parameters set: $\gamma=1.5, \varepsilon=0.15$, and $\delta=1.2$. (a) $t=0$; (b) $t=100$; (c) $t=200$; (d) $t=300 ;$ (e) $t=400$; and (f) $t=500$.

\section{Acknowledgments}

This research was partially supported by the National Natural Science Foundation of China under Grant nos. 11301490, 11301491, 11331009, 11147015, 11171314, 11305043, and 11105024; Natural Science Foundation of Shan'xi Province Grant nos. 2012021002-1 and 2012011002-2, the Opening Foundation of Institute of Information Economy, Hangzhou Normal University, Grant no. PD12001003002003; and the specialized research fund for the doctoral program of higher education (preferential development) Grant no. 20121420130001.

\section{References}

[1] W. C. Allee, Animal Aggregations, University of Chicago Press, 1931.

[2] W. C. Allee and E. Bowen, "Studies in animal aggregations: mass protection against colloidalsilver among goldfishes," Journal of Experimental Zoology, vol. 61, pp. 185-207, 1932.

[3] W. C. Allee, O. Emerson, T. Park, and K. Schmidt, Principles of Animal Ecology, Saunders, Philadelphia, Pa, USA, 1949.

[4] W. C. Allee, Cooperation Among Animals, Henry Shuman, 1951.

[5] W. C. Allee, The Social Life of Animals, Beacon Press, 1958.

[6] F. Courchamp, T. Clutton-Brock, and B. Grenfell, "Inverse density dependence and the Allee effect," Trends in Ecology and Evolution, vol. 14, no. 10, pp. 405-410, 1999.
[7] P. A. Stephens and W. J. Sutherland, "Consequences of the Allee effect for behaviour, ecology and conservation," Trends in Ecology and Evolution, vol. 14, no. 10, pp. 401-405, 1999.

[8] J. Cushing and J. T. Hudson, "Evolutionary dynamics and strong allee effects," Journal of Biological Dynamics, vol. 6, pp. 941-958, 2012.

[9] S. N. Elaydi and R. J. Sacker, "Population models with Allee effect: a new model," Journal of Biological Dynamics, vol. 4, no. 4, pp. 397-408, 2010.

[10] J. Shi and R. Shivaji, "Persistence in reaction diffusion models with weak Allee effect," Journal of Mathematical Biology, vol. 52, no. 6, pp. 807-829, 2006.

[11] J. Wang, J. Shi, and J. Wei, "Predator-prey system with strong Allee effect in prey," Journal of Mathematical Biology, vol. 62, no. 3, pp. 291-331, 2011.

[12] A. Verdy, "Modulation of predator-prey interactions by the Allee effect," Ecological Modelling, vol. 221, no. 8, pp. 1098-1107, 2010.

[13] V. Méndez, C. Sans, I. Llopis, and D. Campos, "Extinction conditions for isolated populations with Allee effect," Mathematical Biosciences, vol. 232, no. 1, pp. 78-86, 2011.

[14] S. V. Petrovskii, A. Y. Morozov, and E. Venturino, "Allee effect makes possible patchy invasion in a predator-prey system," Ecology Letters, vol. 5, no. 3, pp. 345-352, 2002.

[15] A. Morozov, S. Petrovskii, and B.-L. Li, "Bifurcations and chaos in a predator-prey system with the Allee effect," Proceedings of the Royal Society B, vol. 271, no. 1546, pp. 1407-1414, 2004. 
[16] A. Morozov, S. Petrovskii, and B.-L. Li, "Spatiotemporal complexity of patchy invasion in a predator-prey system with the Allee effect," Journal of Theoretical Biology, vol. 238, no. 1, pp. 18-35, 2006.

[17] G. H. Gunaratne, Q. Ouyang, and H. L. Swinney, "Pattern formation in the presence of symmetries," Physical Review E, vol. 50, no. 4, pp. 2802-2820, 1994.

[18] B. Peña and C. Pérez-García, "Stability of Turing patterns in the Brusselator model," Physical Review E, vol. 64, no. 5, part 2, Article ID 056213, 9 pages, 2001.

[19] J. A. Sherratt, "Periodic travelling waves in cyclic predator-prey systems," Ecology Letters, vol. 4, no. 1, pp. 30-37, 2001.

[20] M. Baurmann, T. Gross, and U. Feudel, "Instabilities in spatially extended predator-prey systems: spatio-temporal patterns in the neighborhood of Turing-Hopf bifurcations," Journal of Theoretical Biology, vol. 245, no. 2, pp. 220-229, 2007.

[21] M. A. Lewis and P. Kareiva, "Allee Dynamics and the Spread of Invading Organisms," Theoretical Population Biology, vol. 43, no. 2, pp. 141-158, 1993.

[22] A. M. Turing, "The chemical basis of morphogenesis," Philosophical Transactions of the Royal Society B, vol. 237, pp. 37-72, 1952.

[23] Q. Ouyang, Pattern Formation in Reaction-Diffusion Systems, Sci-Tech Education Publishing House, Shanghai, China, 2000.

[24] F. Courchamp, J. Berec, and J. Gascoigne, Allee Effects in Ecology and Conservation, Oxford University Press, New York, NY, USA, 2008.

[25] J. D. M. Rademacher, B. Sandstede, and A. Scheel, "Computing absolute and essential spectra using continuation," Physica D, vol. 229, no. 2, pp. 166-183, 2007.

[26] P. Wheeler and D. Barkley, "Computation of spiral spectra," SIAM Journal on Applied Dynamical Systems, vol. 5, no. 1, pp. 157-177, 2006.

[27] M. Pascual, "Diffusion-induced chaos in a spatial predator-prey system," Proceedings of the Royal Society B, vol. 251, no. 1330, pp. 1-7, 1993.

[28] J. A. Sherratt, M. J. Smith, and J. D. M. Rademacher, "Locating the transition from periodic oscillations to spatiotemporal chaos in the wake of invasion," Proceedings of the National Academy of Sciences of the United States of America, vol. 106, no. 27, pp. 10890-10895, 2009.

[29] M. P. Hassell, J. H. Lawton, and R. M. May, "Patterns of dynamical behaviour in single-species populations," Journal of Animal Ecology, vol. 45, pp. 471-486, 1976.

[30] A. A. Berryman and J. A. Millstein, "Are ecological systems chaotic-and if not, why not?" Trends in Ecology and Evolution, vol. 4, no. 1, pp. 26-28, 1989. 


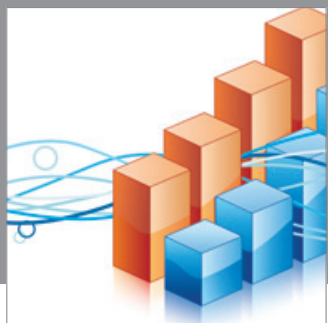

Advances in

Operations Research

mansans

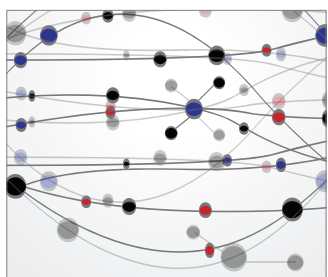

The Scientific World Journal
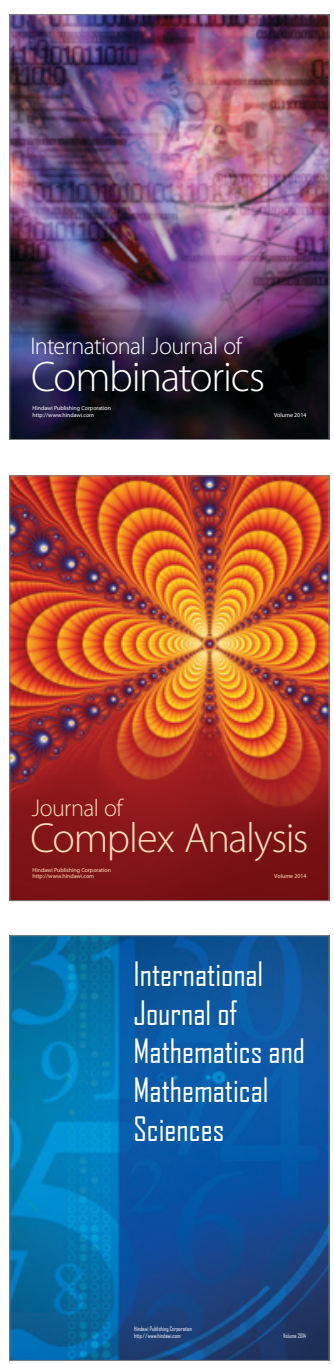
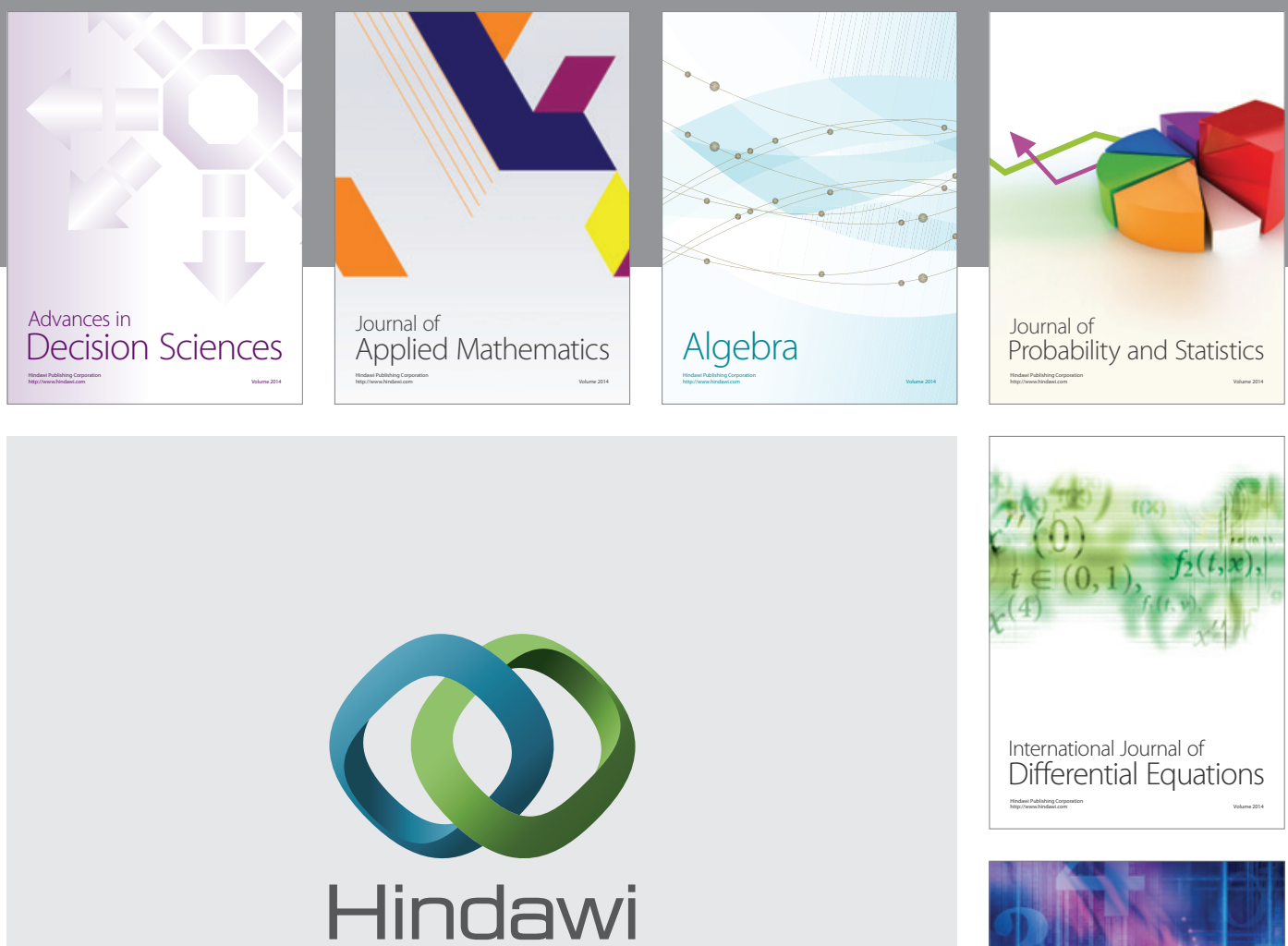

Submit your manuscripts at http://www.hindawi.com
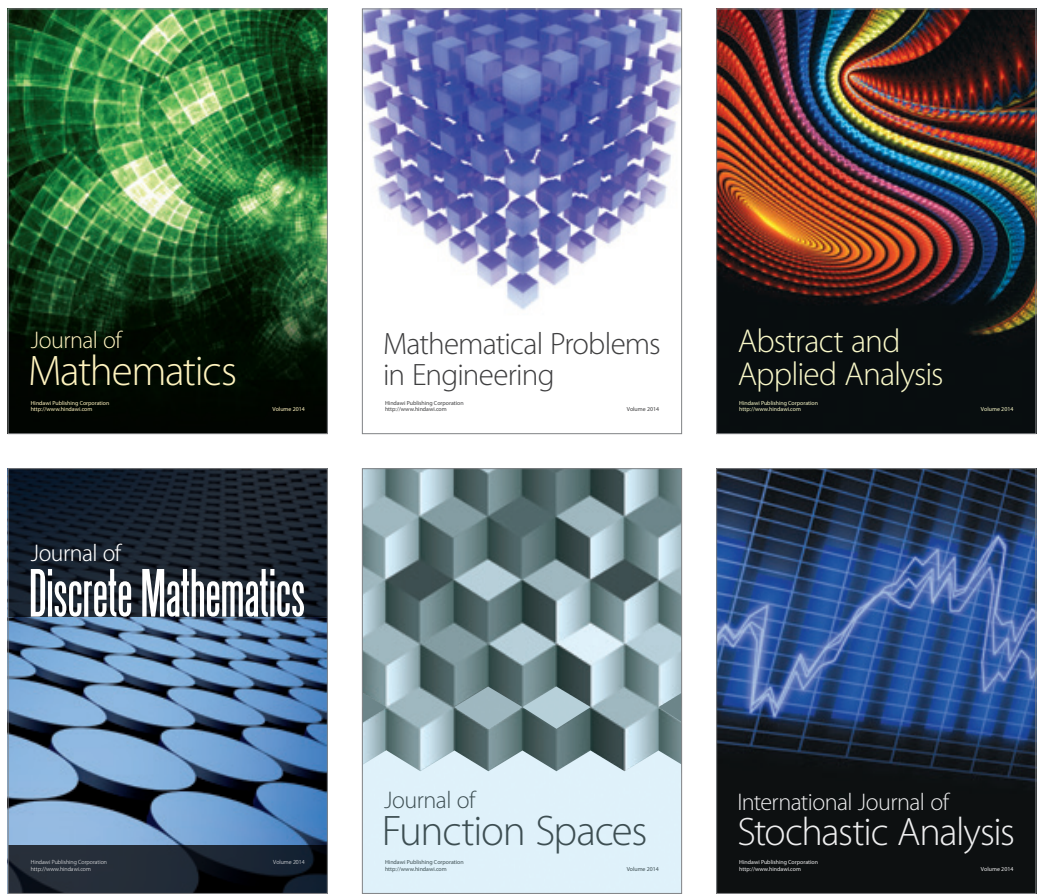

Journal of

Function Spaces

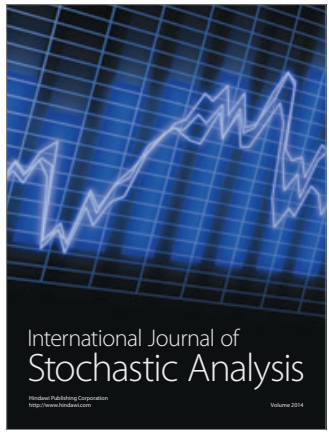

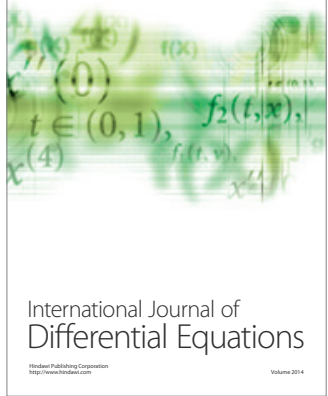
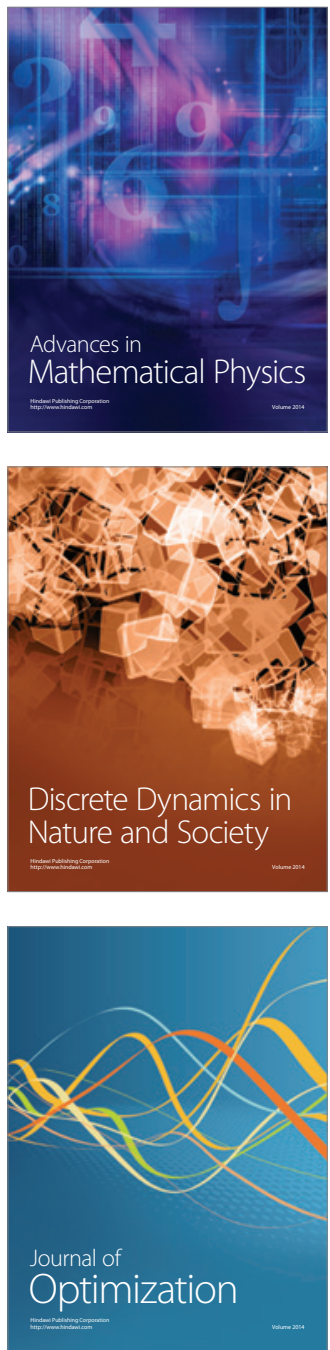